\title{
User Experience of Augmented Reality to Encourage User Satisfaction and Willingness in E-commerce: A Conceptual Framework
}

\author{
Sunisa Junsawang and Singha Chaveesuk ${ }^{+}$ \\ Faculty of Administration and Management, King Mongkut's Institute of Technology Ladkrabang, Bangkok, \\ Thailand
}

\begin{abstract}
The applications of limited augmented reality technology in e-commerce might enhance and create new user's experience. It is apparent that existing e-commerce technology lacks the capability to provide personalized user experience or adequate product information to buyers. Thus, augmented reality technology may enhance the efficiency of e-commerce in respect of purchasing decision, by means of generating virtual information of products. At present, there have been very few studies conducted on the application of augmented reality in e-commerce to improve user experience and, hence, encourage user satisfaction and willingness. In that regard, this paper focuses on constructing a conceptual framework of user experience, satisfaction and willingness by employing augmented reality based on the technology acceptance model. Ultimately, this conceptual framework will provide profound insights into factors that influence user satisfaction and willingness to buy.
\end{abstract}

Keywords: augmented reality, e-commerce, user experience, user satisfaction, user willingness, technology acceptance model

\section{Introduction}

It has been evident that technological innovation has been gaining popularity in the retail industry with the availability of numerous technologies. [1], such as augmented reality (AR) - a technology that allows a computer to overlay physical objects in real time against the computer-generated virtual imagery world. AR enables the user to interact with virtual images using real objects in a seamless way [2]. The implementation of AR in online shopping will improve consumers' shopping experience by displaying product visualizations on images of consumers' physical features, making it a superior e-commerce tool. Indeed, AR has been widely adopted by internationally renowned companies such as L'Oréal and Sephora, which introduced an AR mirror that provides consumers with a virtual makeup experience. Similarly, Nike, Snap, Adidas and eBay have employed various forms of AR, allowing consumers to gain a more vicarious and realistic experience [3]. A previous survey of IKEA's mobile catalogue application, which was conducted based on the technology acceptance model (TAM), found that the perceived usefulness (PU) and perceived ease of use (PEOU) are linked to attitudes (AT) and behavioural intention (BI) towards using the innovation [4]. Moreover, user experience (UX) was found to have an influence on user satisfaction and willingness to buy [5]. Likewise, a previous study suggested that monetary, emotional, convenience and social values are related to hedonic and utilitarian performance expectancies, which subsequently impact usage intention of augmented reality and motion capture (ARMC) e-shopping via a webcam [6]. Furthermore, utilitarian and hedonic shopping values were found to have a positive influence on customer satisfaction [7].

AR e-commerce is anticipated to create new experience for users, owing to the fact that existing ecommerce technology lacks the capability to provide personalized experience or adequate production

+ Corresponding author. Tel.: +662-329-8461.

E-mail address: singha@it.kmitl.ac.th. 
information to buyers. In that regard, AR technology has the potential to improve the efficiency of ecommerce in terms of purchasing decision, by generating virtual information of products to the real world [8]. At present, there have been very few studies conducted on AR e-commerce to improve user experience and, subsequently, user satisfaction and willingness to buy. Thus, this paper focuses on constructing a conceptual framework of user experience, satisfaction and willingness by employing AR based on the technology acceptance model. Ultimately, this conceptual framework will provide profound insights into factors that influence user satisfaction and willingness to buy.

This paper is organized as follows. Section 2 describes the theories and background of this research. Section 3 presents the conceptual framework, relationships, and hypotheses. Finally, Section 4 discusses the contributions and future work of this research.

\section{Theories and Background}

This section describes the theories and background of e-commerce, augmented reality (AR), and technology acceptance model (TAM).

\subsection{E-commerce and augmented reality (AR)}

Electronic commerce (e-commerce) is mode of business and trade market empowered by the internet [9]. Since e-commerce cannot provide sufficient product information to buyers, AR may act as a valuable tool to improve the performance by displaying the virtual products in real-time interface [8]. AR is a technology that can overlay computer generated virtual objects in real scenes or objects. In contrast to virtual reality (VR) that experientially replaces reality, AR upgrades physical reality by incorporating virtual objects into a physical scene, which create virtual objects become, in a sense, an integral part of the real environment [10]. One prominent feature of AR that recognizes it from different types of VR innovation is the media intensity of creating a - mxed reality" wherein the surrounding environment is real, and the objects depicted in nature are virtual. AR upgrades customers' shopping learning by demonstrating product representations on photos of customers' physical features [3].

AR has been introduced to multiple online shopping websites. For example, Chao-Hung et al. [11] conducted a research using Taiwan's famous online shopping website as an experimental platform with embedded AR functions, allowing customers to wear hats that are virtually displayed. The purpose of their research was to evaluate the effectiveness of similar online shopping websites that have not been embedded with AR functions. According to the results, the participants preferred AR to other approaches. In addition, the AR-embedded system was found to reduce mouse clicking times, mouse speed and paging frequency, which implies that AR can facilitate consumers' purchase decision process.

Ana [12] conducted a research to examine consumer behaviour with respect to the implementation of AR in marketing channels, as well as to identify the effects of prior knowledge of interactive technology on consumer behaviour. The results indicated that interactive technology has changed the way consumers engage in shopping activities. Some of the most influential changes attributes to the evolution of web2.0 and web3.0 includes the stakeholders participation in the online community, $\mathrm{B} 2 \mathrm{C}$ and $\mathrm{C} 2 \mathrm{C}$ communications through social media, have also increased adoption of mobile phones and smart applications in our daily lives.

Alternatively, Scott [13] postulated that mobile augmented reality (MAR) application is an increasingly recognized approach that has the potential to enable smart retail. The researcher discussed how MAR applications play a valuable role in the retail industry by creating added value to customers and providing benefits to retailers. The researcher further assessed the level of use, experiential benefits offered, and retail consequences by employing customer experience perspectives in value creation, analysing the content of existing MAR applications, and conducting surveys on smartphone users in the United States.

\subsection{Technology acceptance model (TAM)}

Technology acceptance model (TAM) was introduced by Fred Davis in 1986 for his doctorate proposal. Indeed, TAM is an adaptation of the theory of reasonable actions that is specifically tailored for modelling users' acceptance of information systems or technologies. After that Venkatesh and Davis had found that 
perceived usefulness and perceived ease of use have a direct influence on behavioural intention, thus eliminating the need for the attitude construct [14] as shown in Fig. 1.

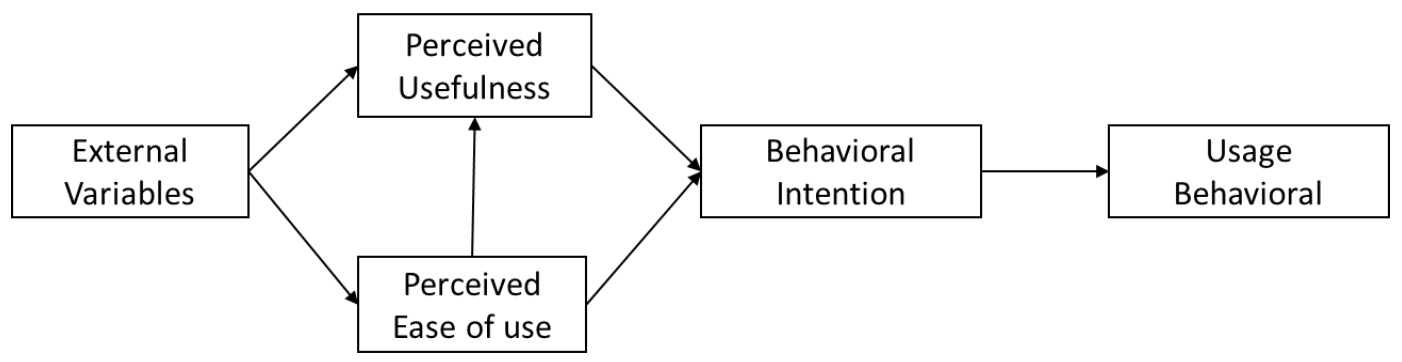

Fig. 1: Technology Acceptance Model (TAM)

According to the research conducted by Muhammad and Dhewanto [15], the Kaskus online e-commerce portal in Indonesia achieved a total transaction of up to 575 million rupiah per month in 2012, reflecting the importance of e-commerce. The research aimed at examining factors the adoption of e-commerce in Indonesia, as well as providing recommendations on future improvements. The collection of data from 223 respondents were analysed using the TAM. The results showed that trust positively affects intended use, while perceived ease of use has an insignificant effect on intended use and risk does not negatively affect intended use. Thus, it can be concluded that the power of existing communities must be maintained, as it has proven to be the key success factor for e-commerce websites.

Another research conducted by Kalina and Marina [16] analysed factors that determine online shopping adoption among young people in the Republic of Macedonia. The researchers proposed a TAM-based framework that was extended by applying relevant constructs that are essential for online shopping, specifically trust, website usability, and customer service. The researchers asserted that online shopping has been gaining popularity among youth in the Republic of Macedonia. It has been recognised that, in general, youth are a strong representative sample of today's online population, especially online shoppers in the country.

\section{Conceptual Framework and Relationships and Hypotheses}

This paper focuses on constructing a conceptual framework of user experience, satisfaction and willingness by using augmented reality based on TAM. The purpose of this paper is to provide profound insights into factors that influence user satisfaction and willingness to buy.

\subsection{AR for E-commerce and TAM}

Alexandra et al. [4] suggested that online customer reviews of products and services have become ubiquitous and easily accessible on the internet, which raises the question whether the analysis of online reviews can replace the traditional methods of measuring technology adoption, such as using questionnaires with TAM items. The research attempted to answer this question by employing IKEA's mobile catalogue application as an example. For comparative purposes, data on the acceptance of the said catalogue were collected in four different ways: (1) as answers to the batteries of TAM items; (2) as assignments to the predetermined adjective pairs; (3) as textual likes and dislikes of users; and (4) as publicly available (real) reviews by users. An example of respondents was utilized as a wellspring of information accumulation for (1) - (3), and an online forum for (4). The data were analysed using partial least squares (PLS) for TAM modelling and text mining to pre-process the textual data.

In summary, the aforementioned research proposed that TAM is helpful for predicting the intention of IKEA's mobile application usage, which is similar to AR e-commerce. To enhance the applicability of TAM to mobile applications, external variables have been incorporated into the TAM, such as perceived enjoyment and perceived informativeness that have an influence on PU and PEOU. This is in line with the research conducted by Nurlina et al., which found that confirmation affected both user experience factors of perceived usefulness and perceived enjoyment, as well as satisfaction [17]. Thus, user experience has a 
significant influence on user satisfaction and continuance usage intention. After taking previous studies and relevant literature into consideration, the following hypotheses have been proposed for this research study:

H1. The relationships of the TAM model are valid for AR E-commerce (PEOU-PU, PU-UX, PEOU-UX, UX-US/ UWB).

H2. PI of AR e-commerce has a direct positive effect on PU.

H3. PE of AR e-commerce has a direct positive effect on PU.

\subsection{User experience (UX), user satisfaction (US) and user willingness to buy (UWB)}

User experience (UX) has been generally disseminated and rapidly accepted in the human-computer interaction (HCI) community. Indeed, the immense interest in UX in academia and industries are attributable to the fact that focuses primarily on user cognition and user performance in human-technology interactions [18].

According to Atieh and Arturo [5], user technology has a positive affect on purchaser choices. However, the way and the extent to which it impacts consumer decisions are obscure. Hence, they studied the impact of AR on retail user experience (UX) and its subsequent influence on user satisfaction and willingness to buy. A total of five hypotheses were tested using a lab experiment. Their results showed that AR significantly shapes UX by impinging on various characteristics of product quality, and UX subsequently influences user satisfaction and willingness to buy.

Alternatively, the research conducted by Ju-Young [6] aimed at examining whether monetary, emotional, convenience and social values are related to hedonic and utilitarian performance expectancies, which subsequently impact usage intention of augmented reality and motion capture (ARMC) e-shopping via a webcam. The research further investigated whether inner self-association and psychological exertion moderate the connection between usage intention and performance expectancies. The model was built dependent on the prospect hypothesis and the value attitude behaviour hierarchy consumer decision model. Data were gathered from US online attire customers who were drawn from an online consumer panel. Structural equation modelling was employed to test the proposed model and research hypotheses. The results suggested that utilitarian performance expectancy is positively related to usage intention. Moreover, monetary, convenience, emotional and social values have an indirect influence on usage intention, mediated by utilitarian performance expectancy, and ego involvement and cognitive effort moderate the link between utilitarian performance expectancy and usage intention.

The results of the research conducted by Atieh and Arturo [5] conform to that of Ju-Young [6], which found that flexibility of use, personalization, utilitarian performance expectancy, shopping effectiveness and perceived usefulness have an influence on usage intention. Hedonic performance expectancy refers to the extent to which an individual believes that it is fun to use technology-based services. The research of JuYoung confirms that monetary, emotional, convenience and social values are positively related to utilitarian performance expectancy and have an indirect influence on usage intention, while convenience and emotional values are positively related to hedonic performance expectancy [6]. Likewise, Saowakhon and Singha proposed that utilitarian and hedonic shopping values positively affect customer satisfaction [7]. These previous research findings ultimately lead to the following hypotheses:

H4. UPE is positively related to UX.

H5. HPE is positively related to UX.

H6. MV is positively related to UPE of AR E-commerce.

H7a-b. CV is positively related to (a) UPE and (b) HPE of AR E-commerce.

H8a-b. EV is positively related to (a) UPE and (b) HPE of AR E-commerce. 


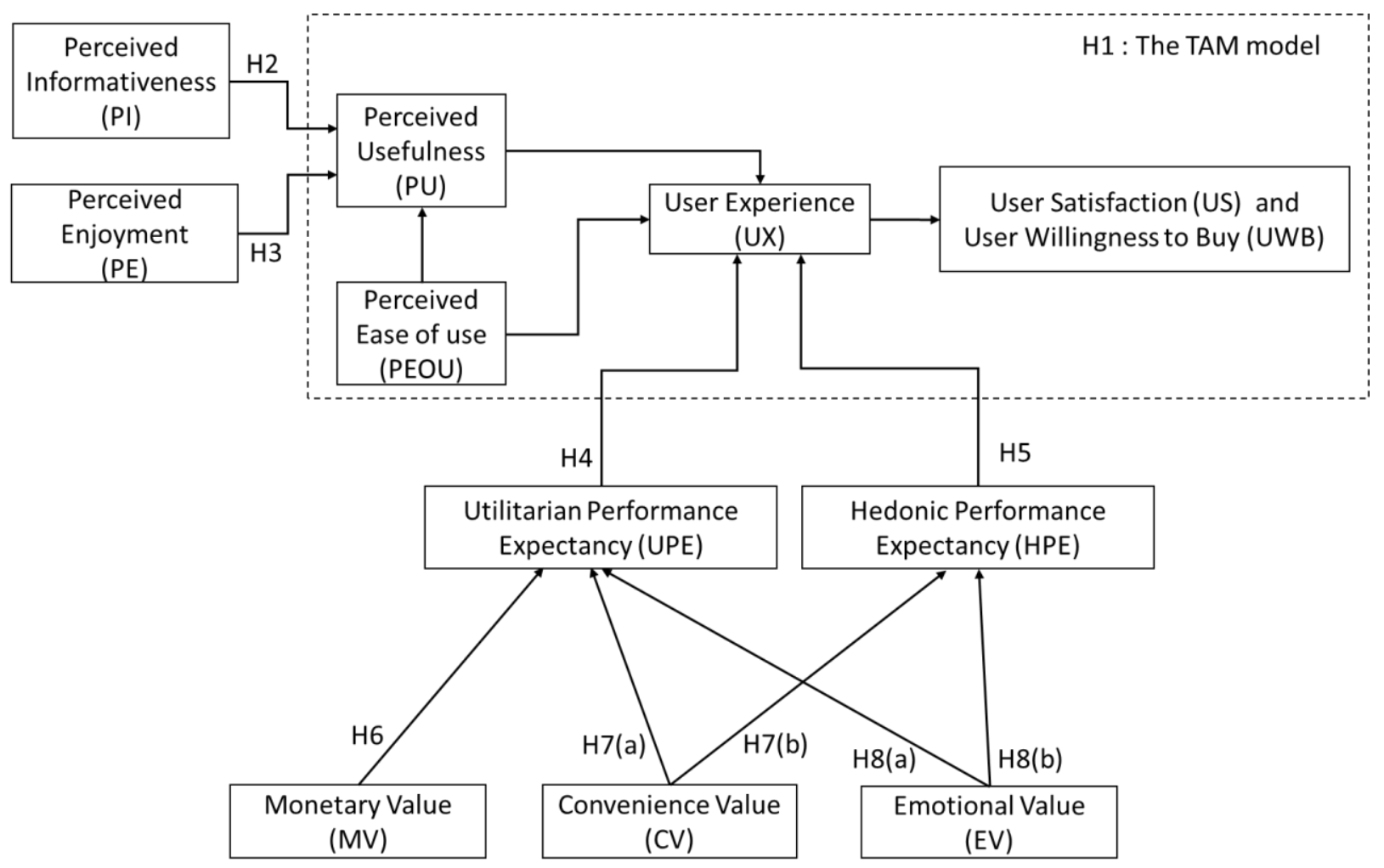

Fig. 2: Conceptual Framework

Fig. 2 represents the TAM-based conceptual framework of user satisfaction and willingness to buy in the context of AR e-commerce and illustrates factors that influence user satisfaction and willingness to buy.

\section{Contributions and Future Work}

This paper proposed a conceptual framework of user experience, satisfaction and willingness to buy by employing augmented reality based on the technology acceptance model. Finally, this conceptual framework provides insights into potential factors that influence user satisfaction and willingness to buy.

For future work, to answer all hypotheses, the quantitative approach will be applied and validate the model using structural equation modelling. However, for the further research, the integration of AR and VR will be examined to improve e-commerce shopping experience on mobile applications.

\section{References}

[1] Alexandra Rese, Daniel Baier, Andreas Geyer-Schulz, and Stefanie Schreiber, "How augmented reality apps are accepted by consumers: A comparative analysis using scales and opinions," Technological Forecasting \& Social Change, pp. 306-319, 2017.

[2] Feng Zhou, Henry Been-Lirn Duh, and M. Billinghurst. Trends in Augmented Reality Tracking, Interaction and Display: A Review of Ten Years of ISMAR [Online].

[3] Mark Yi-Cheon Yim, Shu-Chuan Chu, and P. L. Sauer, "Is Augmented Reality Technology an Effective Tool for E-commerce? An Interactivity and Vividness Perspective," Journal of Interactive Marketing, pp. 89-103, 2017.

[4] Alexandra Rese, Stefanie Schreiber, and D. Baier, "Technology acceptance modeling of augmented reality at the point of sale: Can surveys be replaced by an analysis of online reviews?," Journal of Retailing and Consumer Services, pp. 869-876, 2014.

[5] Atieh Poushneh and A. Z. Vasquez-Parraga, "Discernible impact of augmented reality on retail customer's experience, satisfaction and willingness to buy," Journal of Retailing and Consumer Services, pp. 229-234, 2017.

[6] J.-Y. M. Kang, "Augmented reality and motion capture apparel e-shopping values and usage intention," International Journal of Clothing Science and Technology, 2013. 
[7] Saowakhon Homsud and S. Chaveesuk, "Understanding a Proposed Model of Customer Loyalty Formation in B2C e-Commerce," International Journal of Future Computer and Communication, 2014.

[8] Xinyu Li and D. Chen, "Augmented Reality in E-commerce with Markerless Tracking," presented at the $20102 \mathrm{nd}$ IEEE International Conference on Information Management and Engineering, 2010.

[9] Rima Fayada and D. Paperb, "The Technology Acceptance Model E-Commerce Extension: A Conceptual Framework," presented at the 4th World Conference on Business, Economics and Management, WCBEM, 2015.

[10] Yuzhu Lu and S. Smith, "Augmented Reality E-Commerce Assistant System: Trying While Shopping " Human Computer Interaction, 2007.

[11] Chao-Hung Wanga, Yi-Chen Chianga, and M.-J. Wanga, "Evaluation of an Augmented Reality Embedded On-line Shopping System," presented at the 6th International Conference on Applied Human Factors and Ergonomics (AHFE 2015) and the Affiliated Conferences, AHFE 2015, 2015.

[12] A. Javornik, "Augmented reality: Research agenda for studying the impact of its media characteristics on consumer behaviour," Journal of Retailing and Consumer Services, pp. 252-261, 2016.

[13] S. G. Dacko, "Enabling smart retail settings via mobile augmented reality shopping apps," Technological Forecasting \& Social Change, pp. 243-256, 2017.

[14] P. Lai, "The Literature Review of Technology Adoption Models and Theories for the Novelty Technology," Journal of Information Systems and Technology Management, pp. 21-38, 2017.

[15] Muhammad Luthfihadi and Wawan Dhewanto, "Technology Acceptance of E-commerce in Indonesia," International Journal of Engineering Innovation and Management 32013.

[16] Kalina Trenevska Blagoeva and M. Mijoska, "Applying TAM to Study Online Shopping Adoption Among Youth in the Republic of Macedonia," presented at the Management International Conference 2017, 2017.

[17] Nurlina Setyawan, Muhammad Rifki Shihab, Achmad Nizar Hidayanto, and A. A. Pinem, "Continuance U sage Intention and Intention To Recommend on Information Based M obile Application : A technological and user experience perspective," presented at the 2017 International Conference on Advanced Computer Science and Information Systems (ICACSIS), 2017.

[18] Effie L-C. Law, Virpi Roto, Marc Hassenzahl, Arnold P.O.S. Vermeeren, and J. Kort, "Understanding, Scoping and Defining User eXperience: A Survey Approach," 2009. 